\title{
Anopheles sinensis mosquito insecticide resistance: comparison of three mosquito sample collection and preparation methods and mosquito age in resistance measurements
}

Tielong Xu' ${ }^{1}$ Daibin Zhong ${ }^{2}$, Linhua Tang ${ }^{1}$, Xuelian $\mathrm{Chang}^{3}$, Fengyang $\mathrm{Fu}^{4}$, Guiyun Yan ${ }^{2}$ and Bin Zheng ${ }^{{ }^{*}}$

\begin{abstract}
Background: Insecticide resistance monitoring in malaria mosquitoes is essential for guiding the rational use of insecticides in vector control programs. Resistance bioassay is the first step for insecticide monitoring and it lays an important foundation for molecular examination of resistance mechanisms. In the literature, various mosquito sample collection and preparation methods have been used, but how mosquito sample collection and preparation methods affect insecticide susceptibility bioassay results is largely unknown. The objectives of this study were to determine whether mosquito sample collection and preparation methods affected bioassay results, which may cause incorrect classification of mosquito resistance status.
\end{abstract}

Methods: The study was conducted in Anopheles sinensis mosquitoes in two study sites in central China. Three mosquito sample collection and preparation methods were compared for insecticide susceptibility, kdr frequencies and metabolic enzyme activities: 1) adult mosquitoes collected from the field; 2) F1 adults from field collected, blood-fed mosquitoes; and 3) adult mosquitoes reared from field collected larvae.

Results: Mosquito sample collection and preparation methods significantly affected mortality rates in the standard WHO tube resistance bioassay. Mortality rate of field-collected female adults was 10-15\% higher than in mosquitoes reared from field-collected larvae and F1 adults from field collected blood-fed females. This pattern was consistent in mosquitoes from the two study sites. High kdr mutation frequency (85-95\%) with L1014F allele as the predominant mutation was found in our study populations. Field-collected female adults consistently exhibited the highest monooxygenase and GST activities. The higher mortality rate observed in the field-collected female mosquitoes may have been caused by a mixture of mosquitoes of different ages, as older mosquitoes were more susceptible to deltamethrin than younger mosquitoes.

Conclusions: Female adults reared from field-collected larvae in resistance bioassays are recommended to minimize the effect of confounding factors such as mosquito age and blood feeding status so that more reliable and reproducible mortality may be obtained.

Keywords: Anopheles sinensis, Pyrethroid resistance, Mosquito preparation methods

\footnotetext{
* Correspondence: cdcipdzhengbin@126.com

'National Institute of Parasitic Diseases, Chinese Center for Disease Control and Prevention, and WHO Collaborating Center for Malaria, Schistosomiasis and Filariasis, Ministry of Public Health, Shanghai, China

Full list of author information is available at the end of the article
} 


\section{Background}

Malaria is a main cause of morbidity and mortality worldwide. One important tool to prevent and control malaria is vector control, especially using long-lasting insecticidetreated bed nets (LLINs) and indoor residual spraying (IRS) [1]. Currently, WHO recommends pyrethroids for bed-net impregnation and for indoor residual sprays because of their low toxicity to mammals and humans and high efficacy against mosquitoes [2]. The insecticides used in these public health programs have posed strong selection pressure for resistance. The use of insecticides for agricultural purposes also exerts selection pressure for resistance in mosquitoes because mosquito larvae breed in agricultural fields and thus are directly exposed to insecticides. Furthermore, residual insecticides from agricultural pest control may be leaked into mosquito breeding sites and expose mosquito larvae to insecticides. A key element of resistance management is resistance surveillance.

Resistance bioassay is the first important step in insecticide resistance surveillance. Three sources of mosquitoes have been used for bioassays in the literature: 1) adult mosquitoes collected from the fields were directly used for bioassay, regardless of mosquito age and blood feeding status [3-5]; 2) adult mosquitoes reared from field collected larvae, usually at 3-5 days post emergence [6-8]; and 3) F1 adults from field collected blood-fed mosquitoes, usually at 3-5 days post emergence $[9,10]$. Different mosquito sampling and preparation methods may yield varying results on the knockdown rate and mortality rates because of differences in physiological status (e.g., age and blood feeding status) and possible genetic sampling bias due to small sample size in field mosquito sampling. On the other hand, WHO classifies insecticide resistance status based on bioassay mortality (resistant if mortality rate is $<90 \%$, possible resistance if mortality is between 90 $97 \%$, and susceptible if the rate is $\geq 98 \%$ ) [11]. Therefore, it is important to evaluate the effects of sampling and mosquito preparation methods on the bioassay results so that resistance classification is appropriately conducted. This information will help in understanding the possible bias in bioassay mortality rate and resistance classification resulting from the mosquito sample collection and preparation methods.

Pyrethroid resistance in Anopheles mosquitoes has been studied extensively, primarily in Anopheles gambiae, the most important African malaria vector. Two major resistance mechanisms have been recognized. The first is point mutations in the Para-type sodium channel gene, the target site of pyrethroids, causing a change in affinity between the insecticide and its binding site and leading to knockdown resistance $(k d r)$. The most common mutation conferring $k d r$ is a mutation at position 1014 causing a change from leucine to either phenylalanine (L1014F) or serine (L1014S) [12-19]. However, mutations at other positions of the $k d r$ gene can further enhance pyrethroid resistance (super $k d r$ phenotype) [20,21]. The second mechanism is metabolic detoxification of pyrethroids before they reach their target site by detoxification enzymes, including P450 monooxygenase and glutathione-S-transferases [22-24]. The objective of this study is to determine the effect of mosquito sample collection and preparation methods on resistance bioassays, the $k d r$ gene mutation, and metabolic detoxification enzyme activities.

\section{Methods}

\section{Study sites}

We conducted the study in two sites in China, Liuyang County (three villages, $28.1 \mathrm{~N}$ and 113.4E) in Hunan province and Huainan County (two villages, $32.6 \mathrm{~N}$ and 117.1E) in Anhui province (Figure 1). Malaria in Hunan province was historically endemic, and the latest reported local infection was in 2010. Malaria in the Anhui site is hypoendemic with sporadic vivax malaria outbreaks [25]. Indoor residual spraying in houses surrounding the malaria index case is the main malaria control measure. Rice is the major agricultural crop in these study sites with one harvesting per year. Due to severe insect pest damage on the rice, insecticide use for pest control has been very intensive, with several rounds of sprays in one growing season. Pyrethroids are commonly used for agricultural pest control, but other insecticides, such as organophosphates and carbamates, are also being used [26].

\section{Mosquito sample collection and preparation and resistance bioassay}

The study in Liuyang County, Hunan province was conducted in July and August 2011, and in July and August 2012 for Huainan County, Anhui province. An. sinensis is a predominant vector of malaria in the two study sites. In each study site, three mosquito sample collection and preparation methods were used: 1) adult mosquitoes collected from the fields; 2) F1 adults from field collected, bloodfed mosquitoes; and 3) adult mosquitoes reared from field collected larvae. For the first method, 1,000 female An. sinensis were collected from the pig or cow shelters between 18:00 $\mathrm{h}$ and 21:00 $\mathrm{h}$ using aspirators. After identifying mosquitoes to species morphologically, half of the An. sinensis female mosquitoes were randomly selected for the resistance bioassay, using $0.05 \%$ deltamethrin test paper in the standard WHO tube assay [11]. For the second method, the remaining field-caught and bloodfed mosquitoes (200 mosquitoes) were transferred to a mosquito cage and allowed to lay eggs. Eggs were hatched and larvae were reared in spring water with Tetramin fish food. Immediately after emergence, male and female mosquitoes were separated, and all adults were fed on 10\% sucrose solution. F1 female adults at 3-5 days post emergence were used for the deltamethrin resistance bioassay in the standard WHO 


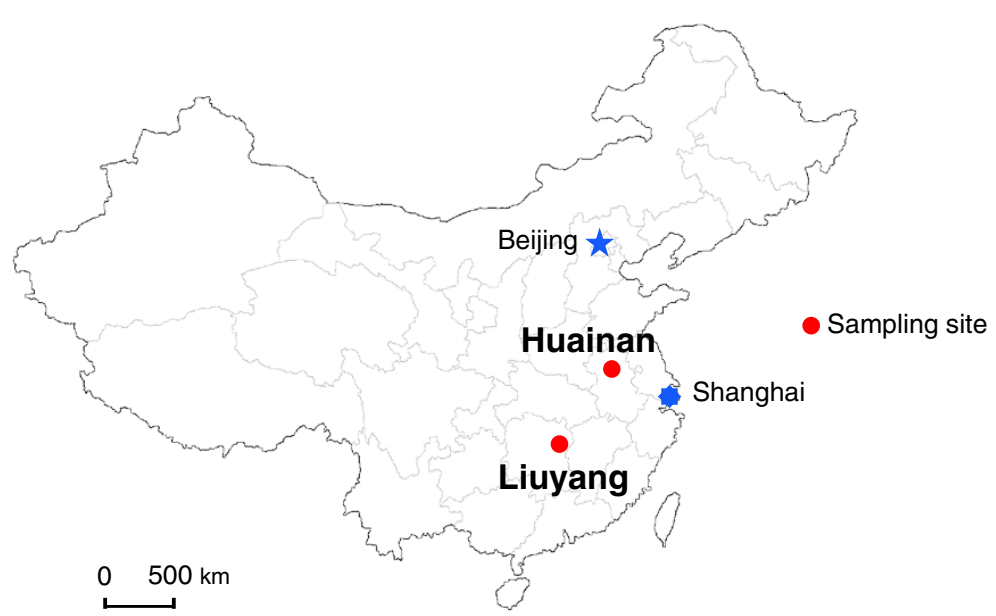

Figure $1 \mathrm{~A}$ map of China showing the distribution of mosquito sampling sites.

tube test [11]. For the third method, adult mosquitoes reared from field-collected larvae, 2,000 larvae from more than 100 larval habitats were collected using the standard $350 \mathrm{ml}$ dippers. The larvae were transported to the local rearing facility and reared to adults, and female adults 3-5 days post emergence were tested for deltamethrin resistance using the standard WHO tube [11]. A laboratory susceptible strain that has been maintained in the insectary of the Chinese Center for Disease Control and Prevention in Shanghai, China, for more than 30 years with no insecticide exposure was used as the reference susceptible strain.

\section{Insecticide susceptibility bioassay}

The female adults from the three sources of mosquitoes were tested for susceptibility to deltamethrin, using the standard WHO tube bioassay with $0.05 \%$ deltamethrin test papers [11]. For each sample collection and preparation method, 140 to 240 female mosquitoes were exposed to WHO papers impregnated with deltemethrin according to WHO protocol, with 20 to 25 mosquitoes per tube. For each sample collection and preparation method, 7-12 replicates were used. Paraffin oil-treated papers without insecticide (control paper) were also tested, and 20 to 25 mosquitoes were used. Mosquitoes were exposed for 1 hour. The knockdown time of individual mosquitoes was recorded every 10 minutes, and the time required for $50 \%$ knockdown of mosquitoes $\left(\mathrm{KT}_{50}\right)$ was determined by the Probit analysis [27] using SAS software. After 1-hour exposure, mosquitoes were transferred to recovery cups and maintained on $10 \%$ sucrose solution for 24 hours and the number of surviving mosquitoes was recorded. All bioassay mosquitoes were tested for metabolic detoxification enzyme activities and a subset of samples were tested for $k d r$ gene mutations.

\section{Effects of mosquito age and blood feeding status on resistance}

The main factors that confound insecticide resistance in field-collected mosquitoes are bloodfeeding status and mosquito age. Therefore, we examined the effects of mosquito age and blood feeding status on insecticide resistance and the underlying mechanisms such as metabolic detoxification enzyme activities. To test the effects of mosquito age, larvae were collected from Huainan county, Anhui province, and reared to adults. Three-day old $(\mathrm{n}=240)$ and 20-day old $(n=45)$ non-blood-fed female adults were bioassayed for resistance to deltamethrin using the standard WHO resistance tube assay described above, and the number of mosquitoes that died after the 24-hour recovery period was recorded. To determine the effects of mosquito blood feeding on resistance, field collected female adults in each site, regardless of blood feeding status, were bioassayed for resistance to deltamethrin. The feeding status of each mosquito was then determined, and the survival status after the 24-hour recovery period was recorded. A mosquito is classified as "blood-fed" if she was fully engorged, and as "non-bloodfed" if the stomach did not contain any blood by visual inspection. A total of 240 non-bloodfed and 80 blood-fed mosquitoes were bioassayed. Thirty mosquitoes were selected randomly from each group for enzyme activity and $k d r$ mutation detecting.

\section{Metabolic enzyme activity assays}

We followed the previously published protocol to measure the activity of glutathione s-transferase (GST) and monooxygenase [12,28,29]. Briefly, individual females were homogenized in $200 \mu \mathrm{l}$ of $\mathrm{KPO}_{4}$ buffer $(0.25 \mathrm{M}$, pH 7.2) and then diluted by adding phosphate buffer. The tube was mixed, centrifuged, and the supernatant was used to test 
GST and monooxygenase. All assays were carried out in duplicate.

\section{Molecular identification and detection of $k d r$ mutation} One leg of each mosquito was used for DNA extraction with the Fast Tissue-to-PCR Kit (Fermentas, CA). Briefly, the mosquito leg was placed at the bottom of a $500 \mu \mathrm{l}$ Eppendorf tube. A total of $50 \mu \mathrm{l}$ of tissue lysis solution and $5 \mu$ of protein $\mathrm{K}$ solution were added and incubated at $55^{\circ} \mathrm{C}$ for $20 \mathrm{~min}$, followed by 10 minutes at $95^{\circ} \mathrm{C}$. After the incubations, $50 \mu \mathrm{l}$ of neutralization solution was added and mixed by vortexing. The neutralized tissue was then centrifuged at $14,000 \mathrm{rpm}$ for $10 \mathrm{~min}$. Extract DNA was stored at $4^{\circ} \mathrm{C}$ or used immediately for PCR. Molecular identifications of $A n$. sinensis species were conducted by using species-specific primers targeting amplification of the ITS2 and 28S rDNA regions (D1 and D2) [30]. To determine point mutations of the $k d r$ gene at position 1014, we amplified a 325 bp fragment, using the primer pair: $k d r$-F TGCCACTCCGTGTGTTTAGA, and $k d r$-R GAG CGATGATGATCCGAAAT. PCR primers were designed based on the An. sinensis sequences of the DIIS6 (domain 2 S6) region of the para-type sodium gene (GenBank acc. no. DQ334052). PCR products were directly sequenced using the big-dye kit by Sangon Biotech CO., Ltd. (Shanghai).

\section{Statistical analysis}

The mortality rate of the mosquitoes exposed to test papers was adjusted by the mortality rate of the mosquitoes in the control group (exposed to paraffin oil-treated papers without insecticide), according to Abbott's formula [11]. To determine the effects of mosquito sample collection and preparation methods, analysis of variance (ANOVA) was conducted using arcsine transformation rate of mosquito mortality from the insecticide susceptibility bioassays and Duncan multiple range tests were used to determine pair-wise differences. One-way ANOVA was also used to examine the difference in monooxygenase and GST activity among the mosquitoes from three sample collection and preparation methods. Statistical significance of differences in mortality between blood-fed and non-bloodfed mosquitoes, or between young (3-day old) and old (20-day old) mosquitoes was examined using the Chi-square test, and the t-test was used to determine the statistical difference in monooxygenase and GST activities. The $k d r$ allele frequency was calculated in each site in each sample collection and preparation method, and between susceptible and resistant mosquitoes. Statistical differences among sample collection and preparation methods were examined using ANOVA, and statistical differences between susceptible and resistant mosquitoes were examined using the Chi-square test.

\section{Ethics statement}

No specific permits were required for the described field studies. For mosquito collection in rice paddies, oral consent was obtained from field owners in each location. These locations were not protected land, and the field studies did not involve endangered or protected species.

\section{Results}

Relationship between mosquito sample collection and preparation methods and bioassay mortality rates

For both the Hunan and Anhui study sites, the mortality rate of field-collected adults was significantly higher than in the adults reared from field-collected larvae and in F1 adults from field-collected blood-fed females (Table 1). For example, in the Anhui study site, a mortality rate of $47.6 \%$ was detected for field-collected adults, significantly higher than the adults reared from field-collected larvae (31.7\%) and F1 adults from field collected bloodfed females (32.9\%). The significantly higher mortality rate in field-collected adults suggests that these individuals were more susceptible to the insecticides, likely due to a mixture of mosquitoes of different ages and various blood feeding statuses within this group. The $\mathrm{KT}_{50}$ varied 92-101 $\mathrm{min}$, and there was no significant difference among the mosquitoes from the three different preparation methods (Table 1). Similar patterns were observed in the Hunan study site (Table 1), although the mortality rates for mosquitoes in all three preparation methods were lower than the Anhui study site, suggesting the Hunan site exhibited higher resistance than the Anhui site. The laboratory susceptible strain showed a mortality rate of $99 \%$. Therefore, both the Hunan and Anhui populations can be classified as "resistant" according to the WHO classification on insecticide resistance [11] because the mortality rates in all three mosquito sample collection and preparation methods were lower than $90 \%$.

\section{Effects of blood feeding status and mosquito age on bioassay mortality}

Blood feeding status had no significant effects on mosquito mortality in the bioassays. The mortality rates of blood-fed and non-bloodfed mosquitoes were $35.4 \%$ and $38.8 \%$, respectively $\left(\chi^{2}=0.45, \mathrm{df}=1 ; P>0.05\right)$ (Table 2$)$. However, mosquito age showed a significant effect, with significantly higher mortality in the 20-day old mosquitoes $(55.7 \%)$ than in the 3 -day old mosquitoes $\left(31.7 \% ; \chi^{2}=\right.$ 9.46, $\mathrm{df}=1 ; P<0.01)$, suggesting older mosquitoes were more susceptible to deltamethrin insecticide.

\section{$K d r$ allele frequencies in An. sinensis populations}

We genotyped a total of 598 mosquitoes collected from the field, including 451 mosquitoes that survived the bioassay (resistant), 147 dead mosquitoes (susceptible), and 50 from the laboratory susceptible colony (Table 3 ). Three 
Table 1 Comparison of mortality rates and knockdown time among three sources of Anopheles sinensis mosquitoes using the standard WHO deltamethrin resistance bioassay

\begin{tabular}{|c|c|c|c|c|c|c|}
\hline \multirow[b]{2}{*}{ Site } & \multirow[b]{2}{*}{ Source of mosquitoes } & \multirow[b]{2}{*}{$\mathbf{n}$} & \multicolumn{2}{|c|}{ Mortality } & \multicolumn{2}{|c|}{ Knockdown time } \\
\hline & & & Mean (\%)* & $\begin{array}{l}95 \% \text { confidence } \\
\text { interval }\end{array}$ & $\mathrm{KT}_{50}(\min )^{*}$ & $\begin{array}{l}\text { 95\% confidence } \\
\text { interval }\end{array}$ \\
\hline \multirow[t]{3}{*}{ Anhui (Huainan county) } & Field-collected female adults & 140 & $47.6^{\mathrm{a}}$ & $42.4-52.8$ & $97^{\mathrm{a}}$ & $81.2-131.5$ \\
\hline & $\begin{array}{l}\text { Female adults reared from } \\
\text { field-collected larvae }\end{array}$ & 240 & $31.7^{\mathrm{b}}$ & $28.7-34.7$ & $92^{\mathrm{a}}$ & $72.3-155.5$ \\
\hline & F1 adults from field blood-fed female & 141 & $32.9^{\mathrm{b}}$ & $27.9-37.9$ & $101^{\mathrm{a}}$ & $83.9-143.9$ \\
\hline \multirow[t]{4}{*}{ Hunan (Liuyang county) } & Field-collected female adults & 180 & $24.9^{\mathrm{a}}$ & $19.2-30.6$ & 104 & $83.4-170.8$ \\
\hline & $\begin{array}{l}\text { Female adults reared from } \\
\text { field-collected larvae }\end{array}$ & 234 & $15.5^{\mathrm{b}}$ & $11.8-19.1$ & - & - \\
\hline & F1 adults from field blood-fed female & 150 & $15.1^{\mathrm{b}}$ & $12.6-17.6$ & - & - \\
\hline & Laboratory strain & 125 & 99.0 & $98.6-99.4$ & 15 & $14.7-16.1$ \\
\hline
\end{tabular}

*For each site, the mortality rate or $\mathrm{KT}_{50}$ that shares different letter indicates statistical difference at $\mathrm{P}<0.05$ using the Duncan multiple range test.

-No mosquitoes were knocked down within the 60-min exposure time, thus $\mathrm{KT}_{50}$ could not be calculated.

$k d r$ alleles at codon 1014 were detected: 1) TTG (wildtype), 2) TGT which causes non-synonymous mutation from leucine (L) to cysteine (C), and 3) TTT and TTC which causes substitution of leucine (L) by phenylalanine (F). We calculated $k d r$ allele frequency for mosquitoes from the three different sample collection and preparation methods for each site (Table 3). Overall, the individuals that we analyzed for $k d r$ in the Hunan and Anhui populations exhibited very high $k d r$ mutation frequencies, with the L1014F and L1014C mutation frequencies exceeding $85 \%$, and the L1014F mutation was the dominant mutation. This is consistent with the previous findings on high $k d r$ mutation frequencies in these study sites [31]. In contrast, the laboratory susceptible population showed very low $k d r$ mutation frequency (1\%). Secondly, the mosquitoes that survived the bioassay (i.e., resistant individuals) consistently showed higher frequencies of mutated $k d r$ alleles than those that died in the bioassay (i.e., susceptible individuals) (Table 3 ), suggesting $k d r$ mutation played a small role in resistance. Overall, the mutated $k d r$ allele frequency in the resistant individuals was $92.1 \%$, significantly higher than the susceptible individuals $(84.0 \%)\left(\chi^{2}=20.4\right.$, $\mathrm{df}=1 ; P<0.001)$.

\section{Metabolic enzyme activities}

We measured P450 monooxygenase and glutathione s-transferase (GST) activities for mosquitoes from three sample collection and preparation methods for each site. In both study sites, field-collected female adults consistently exhibited the highest monooxygenase and GST activities, nearly two fold higher than F1 adults from field blood-fed females for monooxygenase (Figure 2A) and three fold higher for GST (Figure 2B). Bloodfeeding increased GST activity by $95.1 \%(\mathrm{t}=6.54, \mathrm{df}=1, \mathrm{P}<0.001)$ and increased monooxygenase activity by $44.3 \%(\mathrm{t}=6.82$, $\mathrm{df}=1, \mathrm{P}<0.001$ ) (Figure 3A). The 20-day old female mosquitoes exhibited significantly lower GST activity $(\mathrm{t}=6.53$, $\mathrm{df}=1, \mathrm{P}<0.001$ ) and lower monooxygenase activity $(\mathrm{t}=12.28, \mathrm{df}=1, \mathrm{P}<0.001)$ than the 3 -day old female mosquitoes (Figure $3 \mathrm{~B}$ ).

\section{Discussion}

In 2010 China revised its national malaria control strategy and set the goal of malaria elimination by 2020 [32]. Malaria vector control is a key component of the malaria control strategy. However, the rapid rise and spread of insecticide resistance have become major threats to the

Table 2 Effects of blood-feeding status and mosquito age on insecticide resistance bioassay in Anopheles sinensis mosquitoes

\begin{tabular}{|c|c|c|c|c|c|c|}
\hline \multirow[b]{2}{*}{ Trait } & \multirow[b]{2}{*}{ Group } & \multirow[b]{2}{*}{$\mathbf{n}$} & \multicolumn{2}{|c|}{ Mortality } & \multicolumn{2}{|c|}{ Knockdown time } \\
\hline & & & Mortality rate $(\%)^{*}$ & $95 \%$ confidence interval & $\mathrm{KT}_{50}(\mathrm{~min})^{*}$ & $95 \%$ confidence interval \\
\hline \multirow[t]{2}{*}{ Blood feeding status } & Bloodfed & 240 & $35.4^{a}$ & $30.3-40.5$ & - & - \\
\hline & Non-bloodfed & 80 & $38.8^{\mathrm{a}}$ & $33.1-44.5$ & - & - \\
\hline \multirow[t]{2}{*}{ Mosquito age } & 3-day old & 240 & $31.7^{\mathrm{a}}$ & $28.7-34.7$ & $216^{a}$ & $81.4-131.5$ \\
\hline & 20-day old & 45 & $55.6^{b}$ & $52.5-58.7$ & $106^{\mathrm{a}}$ & $61.9-184.5$ \\
\hline
\end{tabular}

*For each trait, the mortality rate that shares different letter indicates statistical difference at $\mathrm{P}<0.05$ using the chi-square test. -Not done. 
Table $3 \mathrm{Kdr}$ allele frequency (in percentage) of Anopheles sinensis mosquitoes among the three sources of mosquitoes used in the bioassay

\begin{tabular}{|c|c|c|c|c|c|c|c|}
\hline Study & Sample preparation & Bioassay & $\mathbf{n}$ & $(\mathrm{TTT}+\mathrm{TTC})$ & L1014C & $\begin{array}{l}\text { Wildtype L1014 } \\
\text { (TTG) }\end{array}$ & $\begin{array}{c}\text { Population } \\
\text { mutation frequency* } \\
(\mathrm{TTT}+\mathrm{TTC}+\mathrm{TGT})\end{array}$ \\
\hline \multirow[t]{6}{*}{ Anhui (Huainan county) } & \multirow{2}{*}{$\begin{array}{l}\text { Female mosquitoes directly collected } \\
\text { from the field }\end{array}$} & Alive & 44 & 81.8 & 14.8 & 3.4 & \multirow[t]{2}{*}{$94.5^{\mathrm{a}}$} \\
\hline & & Dead & 37 & 63.5 & 28.4 & 8.1 & \\
\hline & \multirow{2}{*}{$\begin{array}{l}\text { Female mosquitoes reared from } \\
\text { field-collected larvae }\end{array}$} & Alive & 55 & 78.2 & 19.1 & 2.7 & \multirow[t]{2}{*}{$94.4^{\mathrm{a}}$} \\
\hline & & Dead & 26 & 61.5 & 26.9 & 11.5 & \\
\hline & \multirow{2}{*}{$\begin{array}{l}\text { F1 female from field collected } \\
\text { bloodfed females }\end{array}$} & Alive & 53 & 83.0 & 11.3 & 5.7 & \multirow[t]{2}{*}{$91.2^{\mathrm{b}}$} \\
\hline & & Dead & 27 & 66.7 & 18.5 & 14.8 & \\
\hline \multirow[t]{6}{*}{ Hunan (Liuyang county) } & Female mosquitoes directly & Alive & 89 & 79.2 & 8.4 & 12.4 & \multirow[t]{2}{*}{$84.7^{\mathrm{a}}$} \\
\hline & Collected from the field & Dead & 16 & 62.5 & 6.3 & 31.3 & \\
\hline & \multirow{2}{*}{$\begin{array}{l}\text { Female mosquitoes reared from } \\
\text { field-collected larvae }\end{array}$} & Alive & 121 & 78.5 & 12.0 & 9.5 & \multirow[t]{2}{*}{$88.4^{b}$} \\
\hline & & Dead & 25 & 64.0 & 14.0 & 22.0 & \\
\hline & \multirow{2}{*}{$\begin{array}{l}\text { F1 female from field collected } \\
\text { bloodfed females }\end{array}$} & Alive & 89 & 74.2 & 17.9 & 7.9 & \multirow[t]{2}{*}{$90.5^{b}$} \\
\hline & & Dead & 16 & 56.3 & 25.0 & 19.7 & \\
\hline \multirow[t]{2}{*}{ Total } & & Alive & 451 & 78.6 & 13.5 & 7.9 & \\
\hline & & Dead & 147 & 62.9 & 21.1 & 16.0 & \\
\hline Laboratory strain & & Dead & 50 & 1.0 & 0.0 & 99.0 & 1.0 \\
\hline
\end{tabular}

*For each site, the populations that share different letters indicate statistical difference at $\mathrm{P}<0.05$ using the analysis of molecular variance.

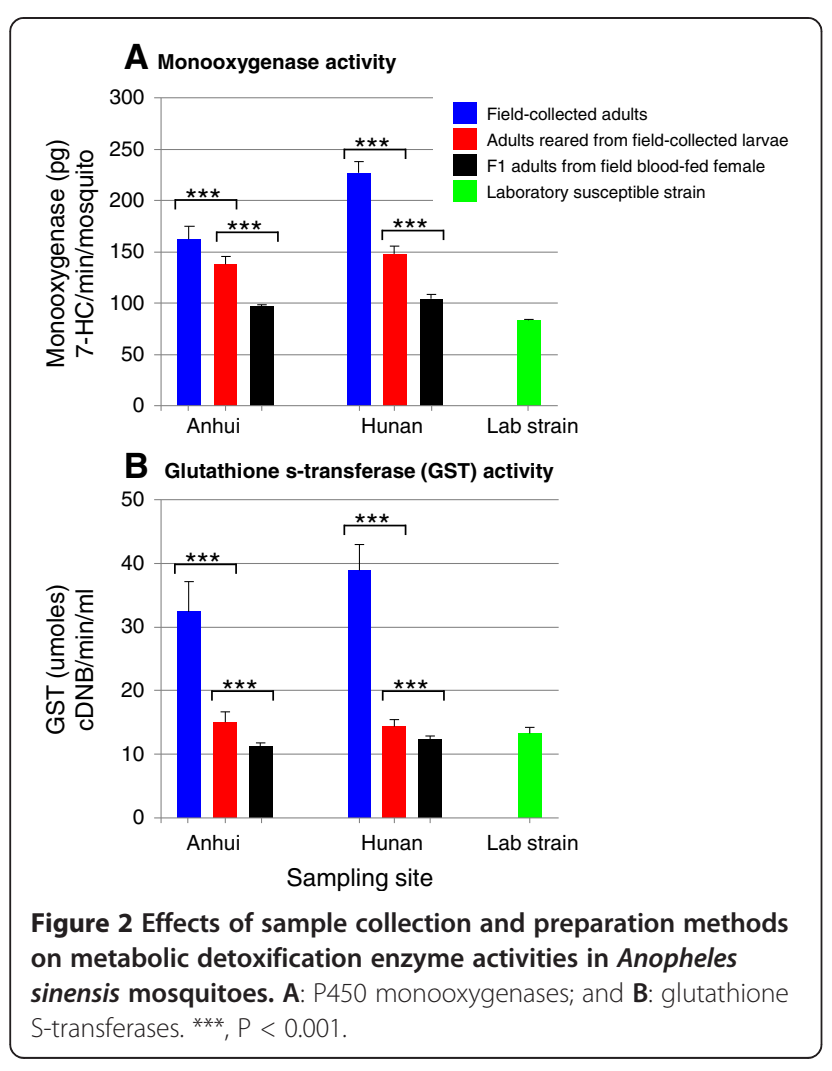

efficiency of insecticide-based vector control activities. Insecticide resistance monitoring in Anopheles mosquitoes is essential to guide the rational use of insecticides in vector control programs. Resistance bioassay with WHO standard tubes or bottles is the first step to quantify insecticide resistance in mosquito populations. At least three mosquito sample collection and preparation methods have been used in the literature, including field-collected adult mosquitoes, F1 adults from blood-fed females collected from the field, and F1 female adults from field-collected larvae as recommended by WHO [11]. Un-resolved issues include: 1) whether mosquito sample collection and preparation methods affect bioassay results, and 2) how much the other sample collection and preparation methods lead to a biased estimation of mosquito mortality rate and thus incorrectly classify the population resistance status. This study was designed to address these two questions using two independent study sites.

We found that mosquito sample collection and preparation methods significantly affected mortality rates in the standard WHO tube resistance bioassay. In particular, the mortality rate of field-collected female adults was the highest, $10-15 \%$ higher than mosquitoes reared from field collected larvae and F1 adults from field blood-fed females. On the other hand, mosquitoes reared from field collected larvae and F1 adults from field blood-fed females exhibited similar mortality rates. This pattern was consistent between the two study sites. Therefore, we conclude that when comparing insecticide resistance across multiple study sites, the same mosquito sample collection and 


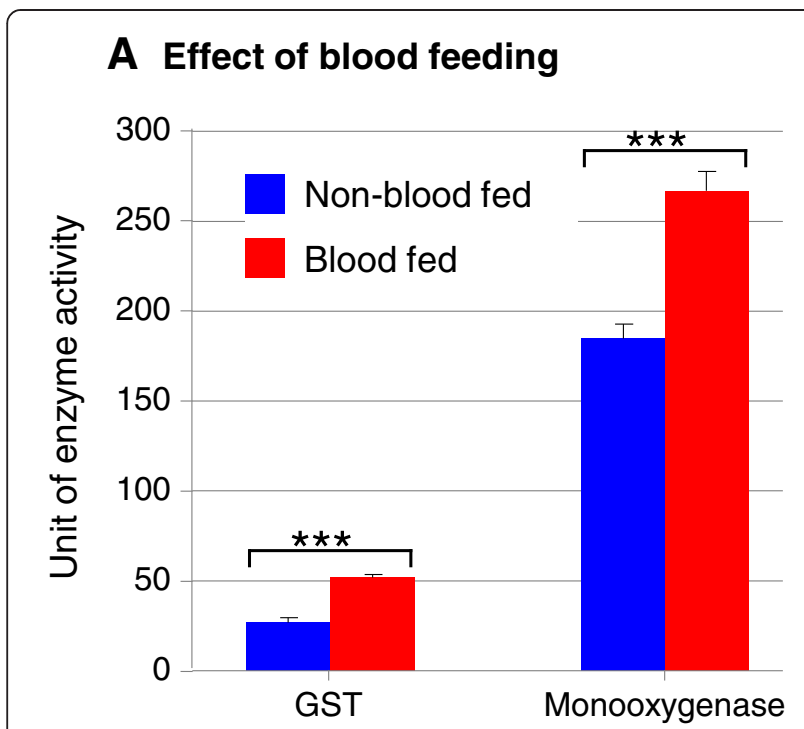

$B$ Effect of mosquito age

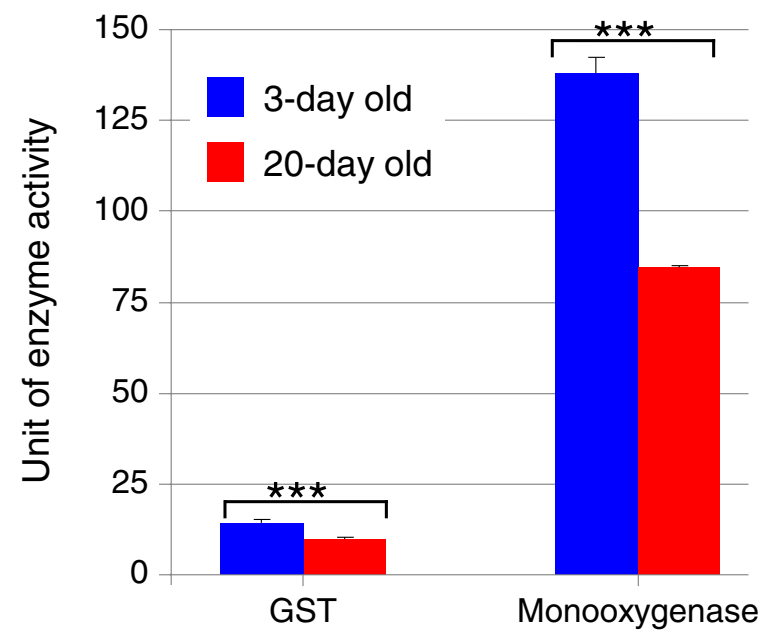

Figure 3 Effects of bloodfeeding (A) and mosquito age (B) on metabolic detoxification enzyme activities in Anopheles sinensis mosquitoes. ${ }^{* *}, P<0.001$.

preparation method must be used, particularly when fieldcollected female mosquitoes are directly used in the bioassay. Because our study populations are highly resistant to deltamethrin, the higher mortality rate detected in fieldcollected female mosquitoes did not lead to incorrect resistance classification based on the WHO standard [11]. However, the $10-15 \%$ higher mortality rate would become critical when mortality rate of the mosquito population is near the threshold level (i.e., $90 \%$ mortality) for resistance classification.

The higher mortality rate observed in the field-collected female mosquitoes is likely to be a result of variability in age. Field-collected female mosquitoes represent a mixture of mosquitoes of different ages and different bloodfeeding history, which may confound the bioassay results
[22,33-35]. In this study we found that older mosquitoes were far more susceptible to deltamethrin than younger mosquitoes. We did not detect significant effects of bloodfeeding on bioassay mortality in this study. Perhaps nonblood-fed mosquitoes may be more active in searching for a bloodmeal and may have a higher chance of being exposed to insecticide-treated nets in natural conditions. However, under our bioassay conditions all mosquitoes were confined to small tubes and all would have a high chance of exposure to the deltamethrin test paper, which may explain why we did not detect significant effects of bloodfeeding on mortality in the susceptibility bioassay.

It is interesting to note that field-collected mosquitoes exhibited the highest monooxygenase and GST activities several folds higher than F1 adults from blood-fed females or female adults reared from field-collected larvae. Such high levels of monooxygenase and GST activities may also be partially caused by the bloodfeeding event because most field-collected females were blood-fed and blood feeding increased GST activity by $95 \%$ and monooxygenase activity by $44 \%$. A recent study that used the stepwise multiple regression analyses in An. sinensis mosquito populations from central and southern China that demonstrated both $k d r$ mutations and monooxygenase activity were significantly associated with deltamethrin resistance, with monooxygenase activity playing a stronger role [31].

\section{Conclusions}

Considering that the field-collected adults could not eliminate the confounding effects of blood feeding and mosquito age and this could likely lead to a biased estimate of insecticide resistance, $\mathrm{F} 1$ female adults reared from field-collected larvae should be used as the first line technique in resistance bioassays because it minimizes the effect of confounding factors such as mosquito age and physiological status so that more reliable and reproducible mortality will be obtained. We do not recommend using field-collected adults in bioassays during resistance monitoring because mixed blood feeding status and mosquito ages in the test specimens likely leads to less reliable and less reproducible mortality estimates. F1 adults from blood-fed females collected from the field may be used for bioassay only when the first line technique is unattainable and when F1 adults are from a large number of field-collected blood-fed females and the founder effect is minimized.

\section{Competing interests}

The authors declare that they have no competing interests.

\section{Authors' contributions}

Conceived and designed the experiments: BZ and GY. Performed the experiments: TX, DZ, XC and FF. Analyzed the data: TX. Wrote and revised the manuscript: TX, LT, BZ and GY. All authors read and approved the final manuscript.

\section{Acknowledgments}

We thank JihuiDuan for assistance with mosquito collection, and the two anonymous reviewers for their valuable comments. This work is supported 
by grants from the National Institutes of Health (R03TW008237, D43TW009527 and U19AI089672). The funders had no role in study design, data collection and analysis, and decision to publish.

\section{Author details}

${ }^{1}$ National Institute of Parasitic Diseases, Chinese Center for Disease Control and Prevention, and WHO Collaborating Center for Malaria, Schistosomiasis and Filariasis, Ministry of Public Health, Shanghai, China. ${ }^{2}$ Program in Public Health, College of Health Sciences, University of California at Irvine, Irvine, CA 92697, USA. ${ }^{3}$ Department of Pathogen Biology, Bengbu Medical College, Anhui, China. ${ }^{4}$ Institute of Entomology and Molecular Biology, College of Life Sciences, Chongqing Normal University, Chongqing, China.

Received: 14 June 2013 Accepted: 17 January 2014

Published: 28 January 2014

\section{References}

1. WHO: Global Malaria Program: Use of indoor residual spraying for scaling up global malaria control and elimination. WHO position statement. Geneva: World Health Organization; 2006. Available at: http://whqlibdoc.who.int/hq/ 2006/WHO_HTM_MAL_2006.1112_eng.pdf?ua=1. Accessed January 17, 2014.

2. WHO: Malaria control today: current WHO recommendations. Geneva: Working document March, World Health Organization; 2005.

3. Hunt R, Edwardes $M$, Coetzee $M$ : Pyrethroid resistance in southern African Anopheles funestusextends to Likoma Island in Lake Malawi. Parasit Vectors 2010, 3:122.

4. Raghavendra K, Verma V, Srivastava HC, Gunasekaran K, Sreehari U, Dash AP: Persistence of DDT, malathion\& deltamethrin resistance in Anopheles culicifacies after their sequential withdrawal from indoor residual spraying in Surat district, India. Indian J Med Res 2010, 132:260-264.

5. Tikar SN, Mendki MJ, Sharma AK, Sukumaran D, Veer V, Prakash S, Parashar BD: Resistance status of the malaria vector mosquitoes, Anopheles stephensi and Anopheles subpictus towards adulticides and larvicides in arid and semi-arid areas of India. J Insect Sci 2011, 11:85.

6. Antonio-Nkondjio C, Fossog BT, Ndo C, Djantio BM, Togouet SZ, Awono-Ambene P, Costantini C, Wondji CS, Ranson H: Anopheles gambiae distribution and insecticide resistance in the cities of Douala and Yaounde (Cameroon): influence of urban agriculture and pollution. Malaria J 2011, 10:154

7. Chandre F, Darrier F, Manga L, Akogbeto M, Faye O, Mouchet J, Guillet P. Status of pyrethroid resistance in Anopheles gambiaesensulato. B World Health Organ 1999, 77(3):230-234

8. Ranson H, Abdallah H, Badolo A, Guelbeogo WM, Kerah-Hinzoumbe C, Yangalbe-Kalnone E, Sagnon N, Simard F, Coetzee M: Insecticide resistance in Anopheles gambiae: data from the first year of a multi-country study highlight the extent of the problem. Malaria J 2009, 8:299.

9. Morgan JC, Irving H, Okedi LM, Steven A, Wondji CS: Pyrethroid resistance in an Anopheles funestus population from Uganda. PloS One 2010, 5(7):e11872.

10. Perera MD, Hemingway J, Karunaratne SP: Multiple insecticide resistance mechanisms involving metabolic changes and insensitive target sites selected in anopheline vectors of malaria in Sri Lanka. Malaria J 2008, 7:168.

11. WHO: Test procedures for insecticide resistance monitoring in malaria vector mosquitoes. Geneva, Switaerland: World Health Organization; 2013.

12. Chen H, Githeko AK, Githure Jl, Mutunga J, Zhou G, Yan G: Monooxygenase levels and knockdown resistance (kdr) allele frequencies in Anopheles gambiae and Anopheles arabiensis in Kenya. J Med Entomol 2008 45(2):242-250

13. Enayati AA, Vatandoost $H$, Ladonni $H$, Townson $H$, Hemingway J: Molecular evidence for a kdr-like pyrethroid resistance mechanism in the malaria vector mosquito Anopheles stephensi. Med Vet Entomol 2003, 17(2):138-144.

14. Kim HBJ, Lee $W-J$, Lee SH: Frequency detection of pyrethroid resistance allele in Anopheles sinensis populations by real-time PCR amplification of specific allele (rtPASA). Pestic Biochem Phys 2007, 87:54-61.

15. Ranson $\mathrm{H}$, Jensen $B$, Vulule JM, Wang $X$, Hemingway J, Collins FH: Identification of a point mutation in the voltage-gated sodium channel gene of Kenyan Anopheles gambiae associated with resistance to DDT and pyrethroids. Insect MolBiol 2000, 9(5):491-497.

16. Singh $\mathrm{OP}$, Dykes $\mathrm{CL}$, Lather $\mathrm{M}$, Agrawal OP, Adak T: Knockdown resistance (kdr)-like mutations in the voltage-gated sodium channel of a malaria vector Anopheles stephensi and PCR assays for their detection. Malaria J 2011, 10:59.
17. Syafruddin D, Hidayati AP, Asih PB, Hawley WA, Sukowati S, Lobo NF: Detection of $1014 \mathrm{~F} \mathrm{kdr}$ mutation in four major Anophelinemalaria vectors in Indonesia. Malaria J 2010, 9:315.

18. Verhaeghen K, Van Bortel W, Roelants P, Backeljau T, Coosemans M: Detection of the East and West African kdr mutation in Anopheles gambiae and Anopheles arabiensis from Uganda using a new assay based on FRET/Melt Curve analysis. Malaria J 2006, 5:16.

19. Verhaeghen K, Van Bortel W, Trung HD, Sochantha T, Keokenchanh K, Coosemans M: Knockdown resistance in Anopheles vagus, An. sinensis, An. paraliaeandAn. peditaeniatuspopulations of the Mekong region. Parasit Vectors 2010, 3(1):59.

20. Jamroz RC, Guerrero FD, Kammlah DM, Kunz SE: Role of the kdr and super-kdr sodium channel mutations in pyrethroid resistance: correlation of allelic frequency to resistance level in wild and laboratory populations of horn flies (Haematobiairritans). Insect Biochem Molec 1998, 28(12):1031-1037.

21. Williamson MS, Martinez-Torres D, Hick CA, Devonshire AL: Identification of mutations in the housefly para-type sodium channel gene associated with knockdown resistance (kdr) to pyrethroid insecticides. Mol Gen Genet 1996, 252(1-2):51-60.

22. Cuamba N, Morgan JC, Irving H, Steven A, Wondji CS: High level of pyrethroid resistance in an Anopheles funestus population of the Chokwe District in Mozambique. PloS One 2010, 5(6):e11010.

23. Munhenga G, Masendu HT, Brooke BD, Hunt RH, Koekemoer LK: Pyrethroid resistance in the major malaria vector Anopheles arabiensis from Gwave, a malaria-endemic area in Zimbabwe. Malaria J 2008, 7:247.

24. Hunt RH, Brooke BD, Pillay C, Koekemoer LL, Coetzee M: Laboratory selection for and characteristics of pyrethroid resistance in the malaria vector Anopheles funestus. Med Vet Entomol 2005, 19(3):271-275.

25. Wang LPJS, Cao WC: The Analysis of Spatio-temporal Character of Malaria Epidemic Situation and Its Determinants. Beijing: Chinese center for disease control and prevention; 2008

26. Cui F, Tan Y, Qiao CL: Filariasis vector in China: insecticide resistance and population structure of mosquito Culex pipiens complex. Pest Manag SC 2007, 63(5):453-458.

27. Finney DJ: Statisical logic in the monitoring of reactions to therapeutic drugs. Meth Inform Med 1971, 10(4):237-245.

28. Brengues C, Hawkes NJ, Chandre F, McCarroll L, Duchon S, Guillet P, Manguin S, Morgan JC, Hemingway J: Pyrethroid and DDT cross-resistance in Aedesaegypti is correlated with novel mutations in the voltage-gated sodium channel gene. Med Vet Entomol 2003, 17(1):87-94.

29. Penilla RP, Rodriguez AD, Hemingway J, Torres JL, Arredondo-Jimenez II, Rodriguez $\mathrm{MH}$ : Resistance management strategies in malaria vector mosquito control. Baseline data for a large-scale field trial against Anopheles albimanusin Mexico. Med Vet Entomol 1998, 12(3):217-233.

30. Joshi D, Park MH, Saeung A, Choochote W, Min GS: Multiplex assay to identify Korean vectors of malaria. Mol Ecol Resour 2010, 10(4):748-750.

31. Zhong D, Chang X, Zhou G, He Z, Fu F, Yan Z, Zhu G, Xu T, Bonizzoni M, Wang $M$, et al: Relationship between Knockdown Resistance (kdr), Metabolic Detoxification and Organismal Resistance to Pyrethroids in Anopheles sinensis. PloS One 2013, 8(2):e55475.

32. China mohotpsro: Action Plan of China Malaria Elimination (2010-2020). Beijing: ministry of health of the people's republic of China; 2010. Available at: http:// whothailand.healthrepository.org/bitstream/123456789/1460/1/Action\%20Plan \%20of\%20China\%20\%20Malaria\%20Elimination\%20_2010-2020_pdf. Accessed January 17,2014

33. Glunt KD, Thomas MB, Read AF: The effects of age, exposure history and malaria infection on the susceptibility of Anopheles mosquitoes to low concentrations of pyrethroid. PloS One 2011, 6(9):e24968.

34. Lines JD, Nassor NS: DDT resistance in Anopheles gambiae declines with mosquito age. Med Vet Entomol 1991, 5(3):261-265.

35. Spillings BL, Coetzee $M$, Koekemoer LL, Brooke BD: The effect of a single blood meal on the phenotypic expression of insecticide resistance in the major malaria vector Anopheles funestus. Malaria J 2008, 7:226.

doi:10.1186/1756-3305-7-54

Cite this article as: Xu et al:: Anopheles sinensis mosquito insecticide resistance: comparison of three mosquito sample collection and preparation methods and mosquito age in resistance measurements. Parasites \& Vectors 2014 7:54. 